\title{
Exact Static Plane Symmetric Soliton-Like Solutions to the Nonlinear Interacting Electromagnetic and Scalar Field Equations in General Relativity
}

\author{
Arnaud Edouard Yamadjako1,2* ${ }^{*}$, Alain Adomou ${ }^{1,3}$, Yélomè J. F. Kpomahou ${ }^{2}$, Jonas Edou ${ }^{1}$, \\ Siaka Massou ${ }^{1}$ \\ ${ }^{1}$ Department of Physics, University of Abomey-Calavi, Abomey-Calavi, Benin \\ ${ }^{2}$ Department of Industrial and Technical Sciences, ENSET-Lokossa, UNSTIM-Abomey, Abomey, Benin \\ ${ }^{3}$ National Higher Institute of Industrial Technology, National University of Sciences, Technologies, Engineering and Mathematics \\ of Abomey, UNSTIM, Abomey, Benin \\ Email: *arnouard@gmail.com
}

How to cite this paper: Yamadjako, A.E., Adomou, A., Kpomahou, Y.J.F., Edou, J. and Massou, S. (2022) Exact Static Plane Symmetric Soliton-Like Solutions to the Nonlinear Interacting Electromagnetic and Scalar Field Equations in General Relativity. Journal of High Energy Physics, Gravitation and Cosmology, 8, 164-177.

https://doi.org/10.4236/jhepgc.2022.81012

Received: September 11, 2021

Accepted: January 3, 2022

Published: January 6, 2022

Copyright $\odot 2022$ by author(s) and Scientific Research Publishing Inc. This work is licensed under the Creative Commons Attribution International License (CC BY 4.0).

http://creativecommons.org/licenses/by/4.0/

\section{Open Access}

\begin{abstract}
This research work is related to soliton solutions considered as models that can describe the complex configuration of elementary particles from the study of the interactions of their fields. It is interested in the interaction of fields between two different elementary particles by expressing their physical properties. For that, we have obtained, exact static plane symmetric soliton-like solutions to the nonlinear equations of interacting electromagnetic and scalar fields taking into account the own gravitational field of elementary particles using the calibrated invariance function $P(I)$. It has been proved that all solutions of the Einstein, nonlinear electromagnetic and scalar field equations are regular with the localized energy density. Moreover, the total charge of particles is finite and the total energy of the interaction fields is bounded. It have been emphasized the importance to the own gravitational field of elementary particles and the role of the nonlinearity of fields in the determination of these solutions. In flat space-time, soliton-like solutions exist but the total energy of the interaction fields is equal to zero. We have also shown that in the linear case, soliton-like solutions are absent.
\end{abstract}

\section{Keywords}

Scalar, Electromagnetic, Gravitational Fields Interaction's, Description of Elementary Particles 


\section{Introduction}

The exact solutions of nonlinear differential equations to describe physical phenomena have become a scientific priority after the development of general relativity (GR) and quantum field theory (QFT). New concepts have been created for this purpose. For example, the strange attractor is linked to the notion of chaos and applied to systems with a low degree of freedom but also the notion of soliton. The soliton, considered as an exact, regular, localized energy density, finite total energy and stable solution of nonlinear differential equations is widely used in pure science [1]. In particle physics, the soliton-like solution is used to describe the complex internal configuration of elementary particles experimentally proven [2] [3] [4]. Several works related to soliton-like solutions have been done. Shikin [5] [6] has elaborated the theory of solitons in general relativity. Bronnikov et al. [7] generalized the conditions for obtaining soliton-like solutions for the interaction equation of the electromagnetic and scalar fields in the presence of the own gravitational field of elementary particles in different symmetries. They showed the existence of soliton solutions with trigonometric and polynomial nonlinearity in the static spherical symmetric metric. Rybakov et al. [8] determined exact solutions of scalar and electromagnetic field equations in the static spherical and cylindrical metric. The results of their work prove that with the calibrated invariance function $P(I)=P_{0}(\lambda I-N)^{2}$, soliton-like solutions are obtained under the condition $\lambda I>N$. Kulyabov et al. [9] established the identical solutions to those of [8] in the static plane symmetric metric with the calibrated invariance function $P(I)=(1-\lambda I)^{2}$. Recently Adomou et al. [10] [11] [12] [13] [14] found plane symmetric solutions to the spinor and gravitational field equations in general relativity theory by using any invariants. The aim of this paper is to determine the exact static plane symmetric soliton-like solutions to nonlinear interacting electromagnetic and scalar field equations taking into account the own gravitational field of the elementary particles by using the calibrated invariance function $P(I)=P_{0}(N-\lambda I)^{2}$ under the conditions $N>\lambda I, P(I)=0$ in $x=0$, while specifying the width and depths of localization of the energy density.

To answer this aim, Section 2 addresses the model and fundamental equations. In Section 3, the solutions of Einstein's equation and of interacting nonlinear electromagnetic and scalar fields are established by taking into account the own gravitational field of the elementary particles. Section 4, gives an account of the influence of the own gravitational field of the elementary particles and the role of the nonlinearity of the fields on the obtained solutions. A conclusion follows in Section 5.

\section{Model and Fundamental Equations}

In general relativity, the Einstein equation has the form:

$$
G_{\mu}^{v}=-\chi T_{\mu}^{v}
$$


where $G_{\mu}^{\nu}$ is Einstein's tensor; $\chi$ is Einstein's gravitation constant and $T_{\mu}^{v}$ represents the energy-momentum metric tensor.

The static plane symmetric metric is chosen in the form [5]:

$$
\mathrm{d} s^{2}=\mathrm{e}^{2 \gamma(x)} \mathrm{d} t^{2}-\mathrm{e}^{2 \alpha(x)} \mathrm{d} x^{2}-\mathrm{e}^{2 \beta(x)}\left(\mathrm{d} y^{2}+\mathrm{d} z^{2}\right)
$$

where the functions $\alpha, \beta$ and $\gamma$ depend only on the spatial variable $x$ and verify the coordinate condition [15]:

$$
\alpha=2 \beta+\gamma .
$$

From (1), (2) and (3), we find the components of Einstein's tensor equation [16]:

$$
\begin{gathered}
G_{0}^{0}=\mathrm{e}^{-2 \alpha}\left(2 \beta^{\prime \prime}-2 \gamma^{\prime} \beta^{\prime}-\beta^{\prime 2}\right)=-\chi T_{0}^{0} \\
G_{1}^{1}=\mathrm{e}^{-2 \alpha}\left(2 \gamma^{\prime} \beta^{\prime}+\beta^{\prime 2}\right)=-\chi T_{1}^{1} \\
G_{2}^{2}=\mathrm{e}^{-2 \alpha}\left(\beta^{\prime \prime}+\gamma^{\prime \prime}-2 \gamma^{\prime} \beta^{\prime}-\beta^{\prime 2}\right)=-\chi T_{2}^{2} \\
G_{2}^{2}=G_{3}^{3} \\
T_{2}^{2}=T_{3}^{3}
\end{gathered}
$$

Let us choose the Lagrangian [17]:

$$
L=\frac{R}{2 \chi}-\frac{1}{4} F_{i j} F^{i j}+\frac{1}{2} \varphi_{, i} \varphi^{i} \psi(I)
$$

where $I=A_{i} A^{i}$ is the chronometric invariant; $\psi(I)=1+\lambda \phi(I)$ is some arbitrary function characterizing the interaction between the nonlinear electromagnetic and scalar fields; $A_{i}\left(A_{0}(x), 0,0,0\right)$ is the 4 -vector potential $\lambda$ represents the parameter of the nonlinearity.

From the Lagrangian (9), we establish the nonlinear scalar and the electromagnetic field equations [8]:

$$
\begin{gathered}
\frac{1}{\sqrt{-g}} \frac{\partial}{\partial x^{v}}\left[\sqrt{-g} g^{v \mu} \varphi_{, \mu} \psi(I)\right]=0 \\
\frac{1}{\sqrt{-g}} \frac{\partial}{\partial x^{\mu}}\left[\sqrt{-g} F^{v \mu}\right]-\varphi_{, i} \varphi^{, i} \psi_{I}(I) A^{v}=0 .
\end{gathered}
$$

In the absence of a current source and a magnetic monopole, the chronometric invariant $I$ is written:

$$
I=A_{0} A^{0}=\mathrm{e}^{-2 \gamma} A_{0}^{2} .
$$

The scalar field Equation (10) is reduced to:

$$
\frac{\mathrm{d}}{\mathrm{d} x}\left[\varphi^{\prime}(x) \psi(I)\right]=0,
$$

which has the solution:

$$
\frac{\mathrm{d} \varphi}{\mathrm{d} x}=\frac{C}{\psi(I)}=C P(I)
$$


On the other hand, the nonlinear electromagnetic field Equation (11) develops into four related equations:

$$
\left\{\begin{array}{l}
\left(\mathrm{e}^{-2 \gamma} A_{0}^{\prime}\right)^{\prime}-\mathrm{e}^{-2 \gamma} P_{I}(I) A_{0}=0, \\
A_{1}=0, \\
\left(\mathrm{e}^{-2 \beta} A_{2}^{\prime}\right)^{\prime}-\mathrm{e}^{-2 \beta} P_{I}(I) A_{2}=0, \\
\left(e^{-2 \beta} A_{3}^{\prime}\right)^{\prime}-\mathrm{e}^{-2 \beta} P_{I}(I) A_{3}=0,
\end{array}\right.
$$

Focusing exclusively on the electric part of the electromagnetic field, the differential equation system (14) reduces to:

$$
\left(\mathrm{e}^{-2 \gamma} A^{\prime}\right)^{\prime}-C^{2} \mathrm{e}^{-2 \gamma} P_{I}(I) A=0, \quad A_{0}(x)=A(x) .
$$

The energy-momentum metric tensor of interacting nonlinear electromagnetic and scalar fields is:

$$
T_{\mu}^{v}=\varphi_{, \mu} \varphi^{v} \psi(I)-F_{\mu i} F^{v i}+\varphi_{, i} \varphi^{i} \psi_{I}(I) A^{v} A_{\mu}-\delta_{\mu}^{v}\left[-\frac{1}{4} F_{i j} F^{i j}+\frac{1}{2}\left(\varphi_{, i} \varphi^{i}\right) \psi(I)\right] .
$$

Its non-zero components verify the equalities:

$$
\begin{gathered}
T_{0}^{0}=\frac{1}{2}\left[\mathrm{e}^{-2 \gamma}\left(A^{\prime}\right)^{2}+C^{2} P(I)+2 \mathrm{e}^{-2 \gamma} C^{2} P_{I}(I)(A)^{2}\right], \\
T_{1}^{1}=-T_{2}^{2}=-T_{3}^{3}=\frac{1}{2} \mathrm{e}^{-2 \alpha}\left[-C^{2} P(I)+\mathrm{e}^{-2 \gamma}\left(A^{\prime}\right)^{2}\right] .
\end{gathered}
$$

The sum of Einstein's tensors $\left(\begin{array}{l}1 \\ 1\end{array}\right)+\left(\begin{array}{l}2 \\ 2\end{array}\right)$, leads to:

$$
\beta^{\prime \prime}+\gamma^{\prime \prime}=0 \text {, }
$$

which has the solution:

$$
\beta(x)=-\gamma(x)+E x+D, \quad E=\text { const }, \quad D=\text { const. }
$$

Summation of Einstein's tensors $\left(\begin{array}{l}0 \\ 0\end{array}\right)+\left(\begin{array}{l}1 \\ 1\end{array}\right)$, we find:

$$
\beta^{\prime \prime}=-\frac{1}{2} \chi\left(A A^{\prime} \mathrm{e}^{-2 \gamma}\right)^{\prime} .
$$

Substituting (21) into (19), we can rewrite (19) in the form:

$$
\gamma^{\prime \prime}=\frac{1}{2} \chi\left(A A^{\prime} \mathrm{e}^{-2 \gamma}\right)^{\prime} \text {. }
$$

The first integration of (22) gives:

$$
\gamma^{\prime}(x)=\frac{1}{2} \chi\left(A A^{\prime} \mathrm{e}^{-2 \gamma}\right)+K, \quad K=\text { const. }
$$

For $K=0$, the solution of (23) is:

$$
\mathrm{e}^{2 \gamma}=\frac{\chi A^{2}}{2}+H, \quad H=\text { const. }
$$

The Equation (15) has a generalized solution: 


$$
\pm \frac{C}{\sqrt{H}}\left(x+x_{1}\right)=\int \frac{\mathrm{d} A}{\left(\frac{\chi A^{2}}{2}+H\right) \sqrt{P(I)}}, \quad x_{1}=\text { const. }
$$

The energy density per unit invariant volume, $T(x)=T_{0}^{0} \sqrt{{ }_{-}^{3} g}$ is:

$$
T(x)=\frac{1}{2}\left[C^{2} P(I)+C^{2} \frac{P(I)}{H} \mathrm{e}^{2 \gamma}+2 I C^{2} P_{I}(I)\right] \mathrm{e}^{-\gamma} .
$$

The total energy $E_{f}$ of interacting nonlinear electromagnetic and scalar fields verifies:

$$
E_{f}=\int_{-\infty}^{+\infty} T(x) \mathrm{d} x
$$

In generally from (11) one gets [9]:

$$
j^{v}=-\varphi_{, i} \varphi^{i} \psi_{I}(I) A^{v} .
$$

where $j^{v}$ is the 4 -vector current density tensor. Its non-zero components are:

$$
\begin{gathered}
j^{0}=-\mathrm{e}^{-2(\gamma+\alpha)} C^{2} P_{I}(I) A, \\
j^{1}=j^{2}=j^{3}=0 .
\end{gathered}
$$

The charge density $\rho_{e}(x)$, the charge density per unit invariant volume $\rho(x)$ and the total charge $Q$ of elementary particles become:

$$
\begin{gathered}
\rho_{e}(x)=-C^{2} \mathrm{e}^{-(2 \alpha+\gamma)} P_{I}(I) A \\
\rho(x)=-C^{2} \mathrm{e}^{-2 \gamma} P_{I}(I) A \\
Q=\int_{-\infty}^{+\infty}-C^{2} \mathrm{e}^{-2 \gamma} P_{I}(I) A \mathrm{~d} x .
\end{gathered}
$$

Let us use in Section 3, the concrete form:

$$
P(I)=P_{0}(N-\lambda I)^{2}
$$

where $P_{0}, N$ are some dimensionless constants satisfy $N>\lambda I$ and $P(I)=1$, $x=0$, to establish the solutions of the Einstein and of the nonlinear interacting electromagnetic and scalar field equations.

\section{Exact Static Plane Symmetric Solutions of the Einstein Equation, the Nonlinear Interacting Electromagnetic and Scalar Field Equations}

From (12), (24), (25) and (32), we obtain the expression of the electric scalar potential:

$$
A(x)=\sqrt{\frac{N H}{\lambda\left(1-\sigma^{2}\right)}} \tanh \left[b\left(x+x_{1}\right)\right],
$$

where $b=C \sqrt{N P_{0} \lambda\left(1-\sigma^{2}\right)}$ and $\sigma^{2}=\frac{\chi N}{2 \lambda}$.

Assuming $E=D=0$, the relations (3), (20), (24) and (33), give the expressions of the non-zero components of the metric tensor: 


$$
g_{00}(x)=\frac{H}{1-\sigma^{2}}\left[1-\frac{\sigma^{2}}{\cosh ^{2}\left[b\left(x+x_{1}\right)\right]}\right]
$$

and

$$
g_{11}(x)=g_{22}(x)=g_{33}(x)=-\frac{1-\sigma^{2}}{H} \frac{\cosh ^{2}\left[b\left(x+x_{1}\right)\right]}{\cosh ^{2}\left[b\left(x+x_{1}\right)\right]-\sigma^{2}}
$$

Figure 1 below, shows numerically the properties of the electric scalar potential $A(x)$ and of the component $g_{00}(x)$ of the metric tensor.

In Figure 1(a) and Figure 1(b):

- The electric scalar potential $A(x)$ is a regular function, tending respectively to:

$$
\begin{gathered}
A(x) \mapsto 0 \quad(x \mapsto 0) \\
A(x) \mapsto \pm \sqrt{\frac{N H}{\lambda\left(1-\sigma^{2}\right)}}(x \mapsto \pm \infty),
\end{gathered}
$$

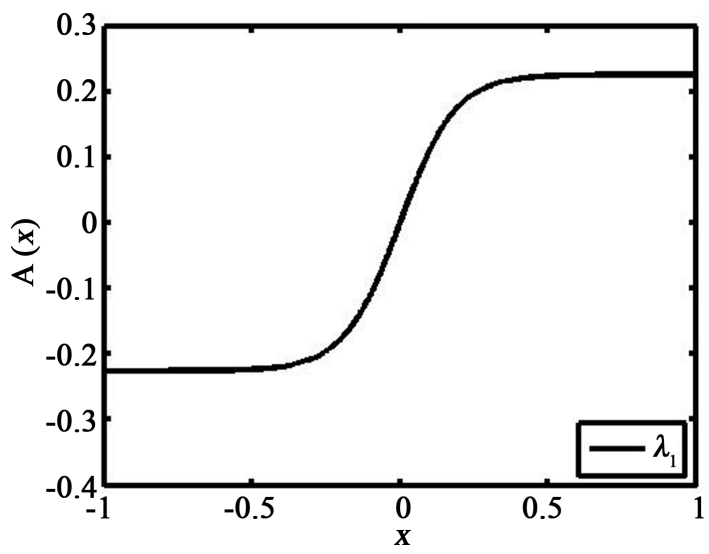

(a)

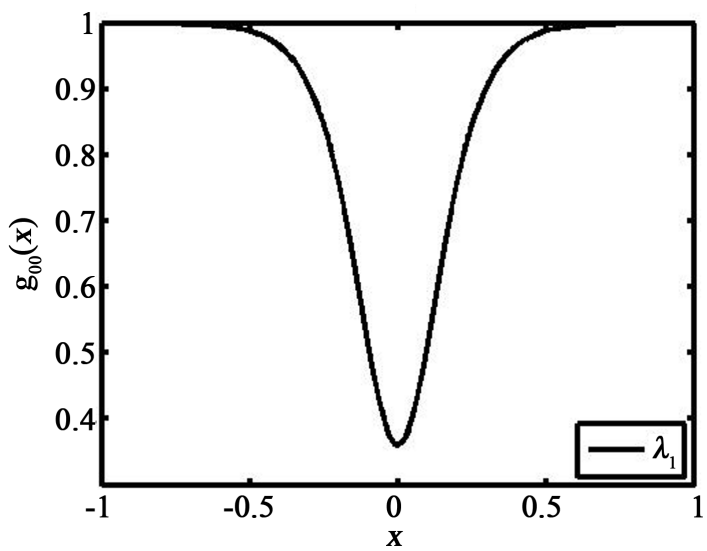

(b)

Figure 1. (a)-Electric scalar potential $A(x)$ and (b)-Component $g_{00}(x)$ of metric tensor. The parameter values used for these simulations are: $\lambda_{1}=39 ; \lambda_{2}=120, x_{1}=0$, $C=N=2$ and $\chi=8 \pi$. 
- The non-zero components of the metric tensor are regular functions taking the values:

$$
\begin{gathered}
g_{00}(x)=-\frac{1}{g_{11}(x)} \mapsto H \quad(x \mapsto 0) \\
g_{00}(x)=-\frac{1}{g_{11}(x)} \mapsto \frac{H}{1-\sigma^{2}} \quad(x \mapsto \pm \infty) .
\end{gathered}
$$

From (33), (34), (35); the relations (12), (17), (26), (29) and (30) become:

$$
\begin{gathered}
I(x)=\frac{N \sinh ^{2}\left[b\left(x+x_{1}\right)\right]}{\lambda\left[\cosh ^{2}\left[b\left(x+x_{1}\right)\right]-\sigma^{2}\right]} ; \\
T_{0}^{0}(x)=M\left[\frac{1-\sigma^{2}-4 \sinh ^{2}\left[b\left(x+x_{1}\right)\right]}{\cosh ^{2}\left[b\left(x+x_{1}\right)\right]\left[\cosh ^{2}\left[b\left(x+x_{1}\right)\right]-\sigma^{2}\right]}\right. \\
\left.+\frac{1}{\cosh ^{4}\left[b\left(x+x_{1}\right)\right]}\right], \\
T(x)=\frac{P\left(1-\sigma^{2}\right) \cosh \left[b\left(x+x_{1}\right)\right]}{\left[\cosh ^{2}\left[b\left(x+x_{1}\right)\right]-\sigma^{2}\right]^{5 / 2}} \\
+\frac{P}{\cosh ^{2}\left[b\left(x+x_{1}\right)\right]\left[\cosh ^{2}\left[b\left(x+x_{1}\right)\right]-\sigma^{2}\right]^{3 / 2}} \\
-\frac{4 P \sinh ^{2}\left[b\left(x+x_{1}\right)\right] \cosh ^{2}\left[b\left(x+x_{1}\right)\right]}{\left[\cosh ^{2}\left[b\left(x+x_{1}\right)\right]-\sigma^{2}\right]^{5 / 2}}, \\
\rho_{e}(x)=\frac{2 C^{2} P_{0} H N^{3 / 2} \sqrt{\lambda} \sinh ^{2}\left[b\left(x+x_{1}\right)\right]}{\left[\cosh ^{2}\left[b\left(x+x_{1}\right)\right]\right] \sqrt{\left[\cosh ^{2}\left[b\left(x+x_{1}\right)\right]-\sigma^{2}\right]}}
\end{gathered}
$$

and

$$
\rho(x)=\sqrt{\frac{\lambda}{H}} \frac{2 C^{2} P_{0}\left[N\left(1-\sigma^{2}\right)\right]^{3 / 2} \sinh \left[b\left(x+x_{1}\right)\right]}{\cosh ^{-1}\left[b\left(x+x_{1}\right)\right]\left[\cosh ^{2}\left[b\left(x+x_{1}\right)\right]-\sigma^{2}\right]^{2}},
$$

where $M=\frac{H C^{2} N^{2} P_{0}}{2}$ and $P=\frac{N^{2} P_{0} C^{2}}{2 \sqrt{H}}\left(1-\sigma^{2}\right)^{3 / 2}$.

Figure 2 is a graphical representation of the energy and charge densities with the same values for parameters in Figure 1.

In Figure 2(a), the energy density is a regular, asymptotic and localized function. The increase of the nonlinearity parameter, decreases its localization width but increases its depth. It varies as follows:

$$
\begin{aligned}
T_{0}^{0}(x) & \mapsto H C^{2} \quad(x \mapsto 0) \\
T_{0}^{0}(x) & \mapsto 0 \quad(x \mapsto \pm \infty)
\end{aligned}
$$

In Figure 2(b), the charge density is a regular, asymptotic, localized function with high depth and low localization width as the nonlinear parameter increases. It takes the values: 


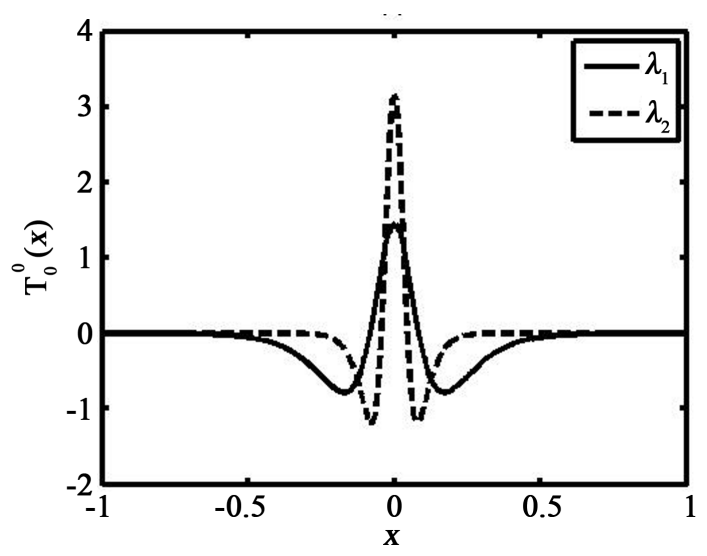

(a)

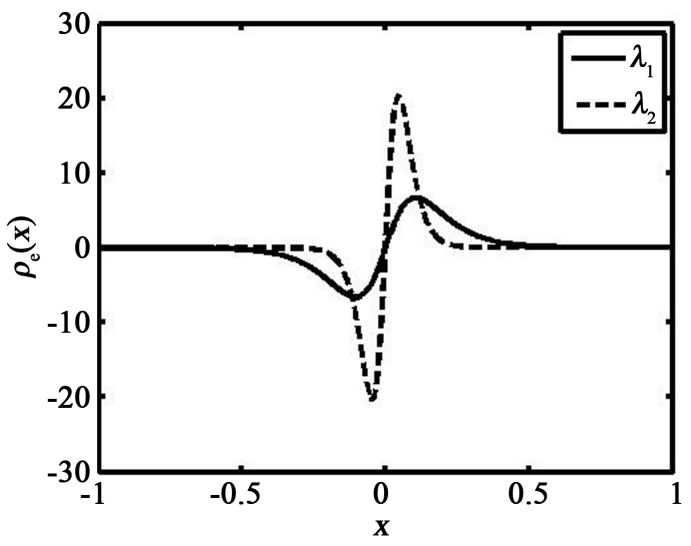

(b)

Figure 2. (a)-Energy density $T_{0}^{0}(x)$ and (b)-Charge density $\rho_{e}(x)$.

$$
\begin{array}{cc}
\rho_{e}(x) \mapsto 0 & (x \mapsto 0) \\
\rho_{e}(x) \mapsto 0 & (x \mapsto \pm \infty)
\end{array}
$$

The charge density per unit volume being an odd function, the charge total of elementary particles (31) is:

$$
Q=0 .
$$

The total energy $E_{f}$ of the interacting nonlinear electromagnetic and scalar fields in the presence of the own gravitational field of the elementary particles verifies:

$$
E_{f}=P\left[\left(1-\sigma^{2}\right) E_{f_{1}}+E_{f_{2}}-4 E_{f_{3}}\right]
$$

where

$$
\begin{gathered}
E_{f_{1}}=\int_{-\infty}^{\infty} \frac{\cosh \left[b\left(x+x_{1}\right)\right]}{\left[\cosh ^{2}\left[b\left(x+x_{1}\right)\right]-\sigma^{2}\right]^{5 / 2}} \mathrm{~d} x \\
E_{f_{2}}=\int_{-\infty}^{\infty} \frac{\mathrm{d} x}{\cosh \left[b\left(x+x_{1}\right)\right]\left[\cosh ^{2}\left[b\left(x+x_{1}\right)\right]-\sigma^{2}\right]^{3 / 2}}
\end{gathered}
$$




$$
E_{f_{3}}=\int_{-\infty}^{\infty} \frac{\sinh ^{2}\left[b\left(x+x_{1}\right)\right] \cosh \left[b\left(x+x_{1}\right)\right]}{\left[\cosh ^{2}\left[b\left(x+x_{1}\right)\right]-\sigma^{2}\right]^{5 / 2}} \mathrm{~d} x
$$

For the determination of the integrals $E_{f_{3}}, E_{f_{2}}$ and $E_{f_{3}}$, we set:

$$
p=\sinh \left[b\left(x+x_{1}\right)\right], u=1+l \sigma^{2}, \quad l=\frac{1}{\cosh ^{2}\left[b\left(x+x_{1}\right)\right]-\sigma^{2}}
$$

and use the following formula from the standard table of integrals [17]:

$$
\begin{aligned}
& \int_{0}^{\infty} \frac{\sinh ^{\mu-1} x \cosh ^{v-1} x}{\left(\cosh ^{2} x-\beta\right)^{\rho}} \mathrm{d} x \\
& =2 B\left(\frac{\mu}{2}, 1+\rho-\frac{\mu+v}{2}\right) \times F\left(\rho, 1+\rho-\frac{\mu+v}{2} ; 1+\rho-\frac{v}{2}, \beta\right) ; \\
& \quad \beta \notin(1, \infty), \operatorname{Re}(\mu)>0,2 \operatorname{Re}(1+\rho)>\operatorname{Re}(v+\mu) .
\end{aligned}
$$

In this expression, $B(a, b)$ is the Bêta function and $F(a, b ; c, m)$ is the Gauss hyper-geometric function. So, we obtain:

$$
\begin{aligned}
E_{f_{1}}= & \frac{4}{3 b\left(1-\sigma^{2}\right)^{2}}\left[1-\frac{\left(1-\sigma^{2}\right) \sinh \left(b x_{1}\right)}{2\left[\cosh ^{2} b\left(x_{1}\right)-\sigma^{2}\right]^{3 / 2}}-\frac{\sinh \left(b x_{1}\right)}{\sqrt{\cosh ^{2} b\left(x_{1}\right)-\sigma^{2}}}\right] ; \\
E_{f_{2}}= & \frac{2}{b \sigma^{2}\left(1-\sigma^{2}\right)}\left[1-\frac{\sinh \left(b x_{1}\right)}{\sqrt{\cosh ^{2} b\left(x_{1}\right)-\sigma^{2}}}\right] \\
& +\frac{1}{b \sigma^{3}}\left[\ln \left|\frac{\sqrt{\cosh ^{2}\left(b x_{1}\right)-\sigma^{2}}+\sigma \sinh \left(b x_{1}\right)}{\sqrt{\cosh ^{2}\left(b x_{1}\right)-\sigma^{2}}-\sigma \sinh \left(b x_{1}\right)}\right|-\ln \left|\frac{1+\sigma}{1-\sigma}\right|\right] ; \\
E_{f} & \frac{1-\sigma^{2}}{2} L\left[\ln \left(\frac{\sqrt{\cosh ^{2}\left(b x_{1}\right)-\sigma^{2}}+\sigma \sinh \left(b x_{1}\right)}{\sqrt{\cosh ^{2}\left(b x_{1}\right)-\sigma^{2}}+\sigma \sinh \left(b x_{1}\right)}\right)-\ln \left(\frac{1+\sigma}{1-\sigma}\right)\right] \\
& +\sigma L\left(1-\frac{{\sinh \left(b x_{1}\right)}^{\cosh ^{2}\left(b x_{1}\right)-\sigma^{2}}}{3 b}\right)
\end{aligned}
$$

where

$$
L=\frac{C N^{3 / 2}}{2 \sigma^{3}} \sqrt{\frac{P_{0}}{H \lambda}} .
$$

Let us now consider the influence of the own gravitational field of the elementary particles and the nonlinearity of the interacting fields in Section 4.

\section{Solutions in Flat Space-Time and in Linear Case}

\subsection{Solutions in Flat Space-Time}

In the absence of the own gravitational field of elementary particles, the metric 
(2) is written:

$$
\mathrm{d} s^{2}=\mathrm{d} t^{2}-\mathrm{d} x^{2}-\mathrm{d} y^{2}-\mathrm{dz} z^{2}
$$

The nonlinear electromagnetic field equation takes the form:

$$
A^{\prime \prime}-C^{2} P_{I}(I) A=0
$$

which has the solution:

$$
\pm C\left(x+x_{1}\right)=\int \frac{\mathrm{d} A}{\sqrt{P(I)}} .
$$

With the calibrated invariance function $P(I)$, the relation (45) leads to:

$$
A(x)=\sqrt{\frac{N}{\lambda}} \tanh \left[v\left(x+x_{1}\right)\right]
$$

where $v=C \sqrt{N P_{0} \lambda}$.

The energy density verify:

$$
T_{0}^{0}(x)=T(x)=\frac{P_{0} N^{2} C^{2}\left[1-2 \sinh ^{2}\left[v\left(x+x_{1}\right)\right]\right]}{\cosh ^{4}\left[v\left(x+x_{1}\right)\right]} .
$$

Figure 3 makes a comparative numerical study between the electric scalar potentials (33) and (46) as well as between the energy densities (37) and (47) obtained respectively in the presence and absence of the own gravitational field of elementary particles.

In Figure 3(a), without the own gravitational field of the elementary particles, the electric scalar potential is a regular function of almost equal amplitude.

In Figure 3(b), the energy density is a regular, asymptotic, localized function. Its depth is more flared and its localization width is almost equal to that obtained in the presence of the own gravitational field of the elementary particles.

The total energy of the interacting nonlinear electromagnetic and scalar fields; the charge density per unit invariant volume and the total charge of elementary particles are given by the expressions:

$$
\begin{gathered}
E_{f}=0, \\
Q=0, \\
\rho(x)=2 C^{2} P_{0} N^{3 / 2} \sqrt{\lambda} \frac{\sinh \left[v\left(x+x_{1}\right)\right]}{\cosh ^{3}\left[v\left(x+x_{1}\right)\right]} .
\end{gathered}
$$

The results obtained in Section 4.1, prove that in the absence of the own gravitational field of the elementary particles, we obtained soliton-like solutions. This soliton-like solution confuses elementary particles with material points. It is opposed to the experimental results obtained in high energy physics. It does not allow to explain the evidence of fermions (leptons and quarks) as well as the gauge bosons and the Higgs boson of the standard model. The gravitational field of the elementary particles plays a role of interaction of the fields and should not be neglected. Let's look at the role of the nonlinearity of the fields. 


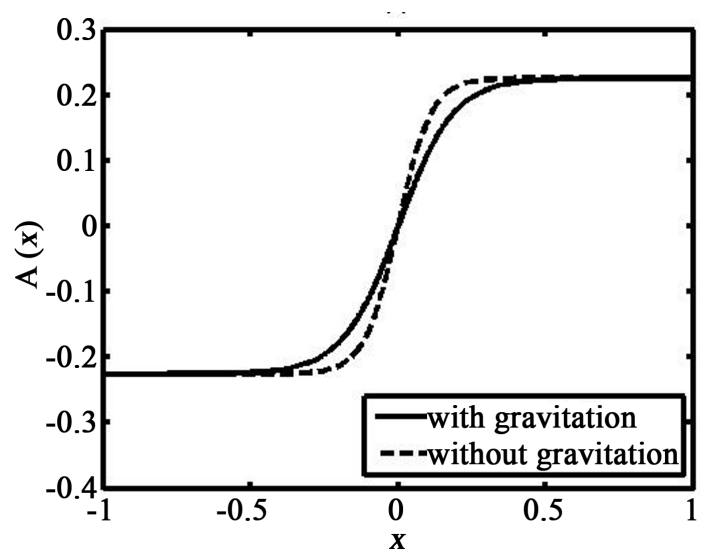

(a)

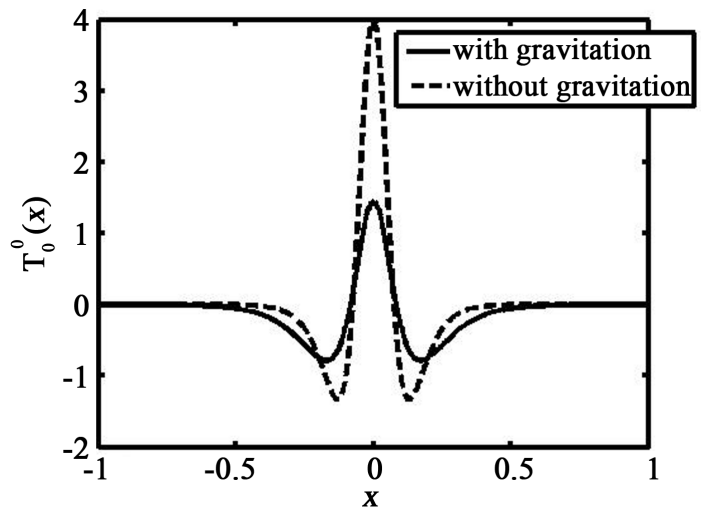

(b)

Figure 3. (a)-Electric scalar potential $A(x)$ and (b)-Energy density $T_{0}^{0}(x)$ keeping the values for the parameters as in Figure 1 and Figure 2 with $\lambda_{1}=\lambda_{2}=39$.

\subsection{Solutions in Linear Case}

In the linear case, the parameter of the nonlinear $\lambda$ is equal to zero. The coupling is minimal and we obtain:

$$
\psi(I)=P(I)=P_{0} N^{2}=1 .
$$

The nonlinear electromagnetic and scalar field equations become:

$$
\begin{gathered}
\left(\mathrm{e}^{-2 \gamma} A^{\prime}\right)^{\prime}=0, \\
\frac{\mathrm{d} \varphi}{\mathrm{d} x}=C .
\end{gathered}
$$

Its lead to solutions:

$$
A(x)=K \int \mathrm{e}^{2 \gamma} \mathrm{d} x
$$

and

$$
\varphi(x)=C x+D .
$$

The energy-momentum metric tensor (16) gives in this case the non-zero components: 


$$
\begin{gathered}
T_{0}^{0}=\frac{1}{2} \mathrm{e}^{-2 \alpha}\left[\mathrm{e}^{-2 \gamma}\left(A^{\prime}\right)^{2}+C^{2}\right] \\
T_{1}^{1}=-T_{2}^{2}=-T_{3}^{3}=\frac{1}{2} \mathrm{e}^{-2 \alpha}\left[\mathrm{e}^{-2 \gamma}\left(A^{\prime}\right)^{2}-C^{2}\right] . \\
\left(\begin{array}{l}
0 \\
0
\end{array}\right)+\left(\begin{array}{l}
1 \\
1
\end{array}\right) \text { and }\left(\begin{array}{l}
1 \\
1
\end{array}\right)+\left(\begin{array}{l}
2 \\
2
\end{array}\right), \text { give respectively: } \\
\beta^{\prime \prime}=-\frac{\chi K^{2}}{2} \mathrm{e}^{2 \gamma}, \\
\gamma^{\prime \prime}=\frac{\chi K^{2}}{2} \mathrm{e}^{2 \gamma} .
\end{gathered}
$$

A solution of (58) is:

$$
\mathrm{e}^{2 \gamma}=-\frac{2 \eta^{2}}{\chi K^{2}} \frac{1}{\cosh ^{2}(\eta x)}, \quad \eta^{2}=\text { const. }
$$

From the relations (3), (54), (57), (58) and (59), we find:

$$
\begin{aligned}
& \mathrm{e}^{2 \alpha}=\mathrm{e}^{2 \beta}=\cosh (\eta x), \\
& A(x)=-\frac{2 \eta}{\chi K} \tanh (\eta x) .
\end{aligned}
$$

The energy density, the energy density per unit invariant volume and the total energy of fields verify the following expressions:

$$
\begin{gathered}
T_{0}^{0}(x)=\frac{1}{2 \cosh ^{2}(\eta x)}\left[C^{2}-\frac{2 \eta^{2}}{\chi} \frac{1}{\cosh ^{2}(\eta x)}\right], \\
T(x)=\frac{1}{2}\left[C^{2} \cosh (\eta x)-\frac{2 \eta^{2}}{\chi} \frac{1}{\cosh (\eta x))}\right], \\
E_{f}=+\infty .
\end{gathered}
$$

The solutions of the Einstein equation, of the electromagnetic and scalar fields are all regular with the localized energy density. The total energy of the fields diverges. These solutions are not soliton-like. The nonlinearity of the fields and the own gravitational field of elementary particles are a determining factor in the description of the complex configuration of the elementary particles.

\section{Conclusion}

The exact static plane symmetric soliton-like solutions for the Einstein equation and the nonlinear electromagnetic and scalar field equation taking into account the own gravitational field of elementary particles have been determined. The obtained results prove that the solutions of the Einstein equation and the nonlinear equation of the interacting electromagnetic and scalar fields are all regular. The energy density is localized. Its width and depth depend on the constant integration and the parameter of the nonlinearity. The total energy of the interacting fields is bounded and the total charge of elementary particles is finite. 
This model is soliton-like solution and can be used to describe the complex internal configuration of elementary particles experimentally proven. It confirms the existence of the elementary particles of the standard model in terms of noncomposite particles. It is opposed to the idea of considering them as material points. When passing to the flat space-time, the total interaction energy of the nonlinear electromagnetic and scalar fields is annulled and the elementary particles are confused with material points. This model does not reflect the real configuration of elementary particles as in the previous case and cannot be used for their description. In linear case, the solutions are not soliton-like. In the following, we will examine the influence of the static symmetric spherical metric.

\section{Conflicts of Interest}

The authors declare no conflicts of interest regarding the publication of this paper.

\section{References}

[1] Scott, A.C., Chu, F.Y.F. and McLaughlin, D.W. (1973) The Soliton: A New Concept in Applied Science. Proceedings of the IEEE, 61, 1443-1483. https://doi.org/10.1109/PROC.1973.9296

[2] Perring, J.K. and Skyrme, T.H.R. (1962) A Model Unified Field Equation. Nuclear Physics, 31, 550-555. https://doi.org/10.1016/0029-5582(62)90774-5

[3] Rybakov, Yu.P. (1985) Particles Structure in Nonlinear Field Theory. Peoples' Friendship University of Russia, Moscow.

[4] Rajaraman, R. (1982) An Introduction to Solitons and Instantons in Quantum Field Theory. North-Holland Publishing Company, New York

[5] Shikin G. N. (1991) Basics of Soliton Theory in General Relativity. URSS Publishers, Moscow

[6] Shikin G. N. (1995) Theory of Solitons in General Relativity. URSS (Undergraduate Research Support Scheme), Moscow.

[7] Bronnikov, K.A., Shikin G.N. (1983) Solitonlike Solutions for Interacting Fields with Consideration of Gravitation. Soviet Physics Journal, 26, 791-794. https://doi.org/10.1007/BF00891841

[8] Rybakov, Yu.P., Shikin, G.N. and Saha, B. (1997) Solitons of Nonlinear Scalar Electrodynamics in General Relativity. International Journal of Theoretical Physics, 36, 1475-1494. https://doi.org/10.1007/BF02435941

[9] Kulyabov, D.J., Rybakov, Yu.P. and Shikin, G.N. (1999) Kink-Like Configurations of Interacting Scalar, Electromagnetic, and Gravitational Fields. Bulletin of Peoples Friendship University of Russia, Series Physics, No. 1, 56-60. http://arxiv.org/abs/math-ph/9902011v1

[10] Adomou, A., Edou, J. and Massou, S. (2019) Plane Symmetric Solutions to the Nonlinear Spinor Field Equations in General Relativity Theory. Journal of Modern Physics, 10, 1222-1234. https://doi.org/10.4236/jmp.2019.1010081

[11] Adomou, A., Alvarado, R. and Shikin, G.N. (1995) Exact Plane-Symmetric Solutions of the Nonlinear Equation of a Spinor Field in the Theory of Gravitation. Russian Physics Journal, 38, 818-824. https://doi.org/10.1007/BF00559285

[12] Adomou, A. and Shikin, G.N. (1997) Exact Self-Consistent Plane-Symmetric of the 
Equation of Two Interacting Fields (Spinor Field and Scalar Field). Russian Physics Journal, 41, 672-678. https://doi.org/10.1007/BF02766486

[13] Adomou, A. and Shikin, G.N. (1999) Exact Self-Consistent Plane-Symmetric Solutions of the Spinor-Field Equation with a Nonlinear Term that Depends on the $S^{2}$ $P^{2}$ Invariant. Russian Physics Journal, 42, 10, 872-877.

https://doi.org/10.1007/BF02523799

[14] Adomou, A. and Shikin G.N. (1999) Exact Self-Consistent Plane-Symmetric Solutions of the Equation for a Spinor Field with a Nonlinear Term that Depends on the Invariant $S^{2}+P^{2}$. Russian Physics Journal, 42, 189-196.

https://doi.org/10.1007/BF02509970

[15] Bronnikov, K.A. and Rubin, S.G. (2013) Black Holes, Cosmology and Extra Dimension. World Scientific Publishing Co., Singapore.

[16] Brill, D. and Wheeler, J. (1985) Cosmology and Extra Dimension. Reviews of Modern Physics, 29, 465-479. https://doi.org/10.1103/RevModPhys.29.465

[17] Bronnikov, K.A. and Shikin, G.N. (1985) On Relativity Theory. In: Choquet-Bruhat, Y. and Karade, T.M., Eds., Proceedings of the Sir Arthur Eddington Centenary Symposium (Vol. 2). World Scientific, Singapore, p. 29, 196. 